\title{
On Shear Wave Speed Estimation for Agar-Gelatine Phantom
}

\author{
Hassan M. Ahmed ${ }^{1,2 *}$ Nancy M. Salem ${ }^{1,}$ Ahmed F. Seddik ${ }^{1,2,}$ Mohamed I. El Adawy ${ }^{1}$ \\ ${ }^{1}$ Biomedical Engineering Department \\ Helwan University \\ Cairo, Egypt \\ ${ }^{2}$ Faculty of Computer Science \\ Nahda University \\ Beni Suef, Egypt
}

\begin{abstract}
Conventional imaging of diagnostic ultrasound is widely used. Although it makes the differences in the soft tissues echogenicities' apparent and clear, it fails in describing and estimating the soft tissue mechanical properties. It cannot portray their mechanical properties, such as the elasticity and stiffness. Estimating the mechanical properties increases chances of the identification of lesions or any pathological changes. Physicians are now characterizing the tissue's mechanical properties as diagnostic metrics. Estimating the tissue's mechanical properties is achieved by applying a force on the tissue and calculating the resulted shear wave speed. Due to the difficulty of calculating the shear wave speed precisely inside the tissue, it is estimated by analyzing ultrasound images of the tissue at a very high frame rate. In this paper, the shear wave speed is estimated using finite element analysis. A model is constructed to simulate the tissue's mechanical properties. For a generalized soft tissue model, Agar-gelatine model is used because it has properties similar to that of the soft tissue. A point force is applied at the center of the proposed model. As a result of this force, a deformation is caused. Peak displacements are tracked along the lateral dimension of the model for estimating the shear wave speed of the propagating wave using the Time-To-Peak displacement (TTP) method. Experimental results have shown that the estimated speed of the shear wave is $5.2 \mathrm{~m} / \mathrm{sec}$. The speed value is calculated according to shear wave speed equation equals about $5.7 \mathrm{~m} / \mathrm{sec}$; this means that our speed estimation system's accuracy is about $91 \%$, which is reasonable shear wave speed estimation accuracy with a less computational power compared to other tracking methods.
\end{abstract}

Keywords-Elasticity Imaging; Acoustic radiation force impulse (ARFI); Shear wave elasticity imaging; Soft tissue stiffness imaging

\section{INTRODUCTION}

Replacing healthy soft tissues by fibrotic tissues is the pathological change that may cause a malignant or benign tumor. The stiffness of these pathologic tissues is higher than the surrounding [1]. Elastic modulus is a measurand for the stiffness. It is the measure of the material's resistance to deformation in either compression or tension namely the elasticity modulus (E) and in shear namely the shear modulus ( $\mu$ ) [2]. Muscles and fibrous tissue are more resistant to deformation than other compliant tissues such as fat, due to their higher elastic moduli [3-5].
Deformation; is a result of stress over the tissue; formerly, it was manual palpation over the tissue. Nowadays, it is the beat or the push that is caused by the acoustic radiation force generated by the ultrasound probe, a procedure called Elastography procedure. This procedure can be accomplished by many methods. These methods are classified regarding either the obtained images or the source of excitation.

Regarding the obtained images, these methods are either qualitative; revealing relative stiffness differences; or quantitative; leading to an estimate for the underlying tissue elastic modulus using reconstruction methods. Also, classification regarding the source of excitation these methods are either external; which are the dynamic external methods and static external compression methods for mechanical excitation; or internal; like the physiological motion of the tissue itself or an acoustic radiation force (ARF).

In this paper, a model is constructed simulating the soft tissue's mechanical properties. The model simulated the Agargelatine phantom, due to its similarity in behavior to soft tissue, where the agar is considered to act as scatterers and gelatin is considered to introduce the elasticity for the model.

A transient ARF is applied to the model to induce scatterers displacements. Then, peak displacements are tracked using Time-To-Peak displacement (TTP) method along the lateral direction starting from the focal zone and moving away towards either of the lateral edges. Speed; of the shear wave generated by the propagation of the peak displacements; is then estimated by calculating the time taken by the wave to reach a specific point on the lateral direction divided by the distance.

This paper is organized as follows, firstly an introduction section about elastography and its major methods, and a background section about the elastography mathematics. Then a literature review section; about the progression in the field of elastography applications starting from its origin till the recent application and the major imaging techniques; is presented. A methodology section demonstrating the FEM generation and ARFI application and acquisition procedure is introduced. Finally, results, discussion, and conclusion sections are given. 


\section{BACKGROUND}

This section aims to give the reader a proper background about the mathematical equations involved in the field of elastography, the assumptions made to simplify the calculations and the limitations that are found if the tissue is modeled as viscoelastic and non-linear.

Firstly, it is worth speaking about the strain, which is the deformation caused by the stress, and to highlight its behavior in the finite element models and its relationship with displacement induced. Strain $(\epsilon)$ is related to the tissue displacement by (1) [1,2], and shown in Figure 1.

$$
\epsilon=\left(\frac{1}{2}\right)\left(\nabla u^{T}+\nabla \mathrm{u}\right)
$$

where $\nabla \mathrm{u}$ is the spatial displacement gradient, and $\mathrm{T}$ is the transpose operation.

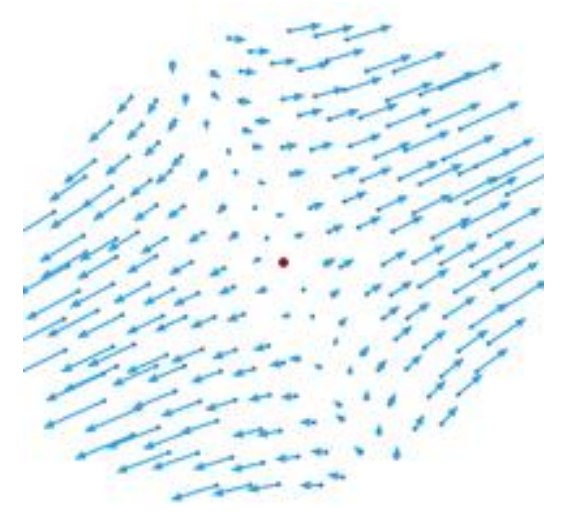

Fig. 1. Spatial displacement gradient

The dynamic displacement response of the soft tissue can be tracked by using the cross-correlation and Doppler-based auto-correlation; such as Kasai's method [6]. The resolution of the tracking methods is not fixed along the lateral and the axial directions. It is better in the axial direction (it is actually fractions of a micrometer) than in the lateral one (a few tens of micrometers) [7]. Axial direction is chosen, because it is the direction of better resolution. Another issue that should be considered is the homogeneity of soft tissues whereas materials constituting them are not homogeneous. Many assumptions are proposed in that field (elasticity imaging field) to simplify the interpretation and the analysis of images obtained. The most common assumptions for the tissue material are $[1,2]$ :

a) Stress-strain relationship of the tissue is linear.

b) The tissue is elastic.

c) The tissue is isotropic.

Under these assumptions we conclude the relation between stress $(\sigma)$ and strain $(\epsilon)$ to be given by (2) [2].

$\sigma=E \epsilon$

where $E$ is the elasticity modulus in $\mathrm{KPa}$.

Another way to estimate the tissue's elastic properties is by tracking the propagation of the shear waves generated inside the tissue. Shear waves propagate orthogonally to the direction of the propagation of the ultrasonic compressive waves; i.e. perpendicular to the direction of the induced tissue displacement.

Under the preceding assumptions of the tissue material, the equation of the shear modulus is given by (3) [1].

$\mu \nabla^{2} u-\rho\left(\frac{\partial^{2} u}{\partial t^{2}}\right)=0$

where $\rho$ is the material density in $\mathrm{Kg} / \mathrm{m}^{3}, \nabla^{2}$ is the Laplacian operator and $\mathrm{t}$ is the time.

The speed of the propagation of the shear wave is also related to the shear modulus according to (4).

$$
C_{x}=\sqrt{\frac{\mu}{\rho}}
$$

Moreover; the shear modulus is related to the elasticity modulus by $(5)[1,2]$.

$$
\begin{aligned}
& \mu= \frac{E}{2(1+v)} \\
& \text { where } v \text { is the Poisson's ratio. }
\end{aligned}
$$

There are two deviations from the above assumptions when modeling the tissue as viscoelastic and non-linear:

a) Considering the viscosity of the tissue results in a dependence of the tissue stiffness on the excitation frequency. Higher excitation frequencies yield a stiffer tissue response compared to lower excitation frequencies. In other words; both the elastic and shear modulus is a function of the frequency $(E(f)$ and $\mu(f))$ [8]. A frequency dependent shear modulus will result in a frequency dependent shear wave speed, a phenomenon called Dispersion.

b) Furthermore, as a result of the introduction of the viscosity term, the tissue would absorb some of the imparted energy.

Nonlinearities imply that the strain in response to an applied stress is dependent on the absolute stress that is applied to the tissue; hence, the elastic moduli are a function of strain $(E(\epsilon)$ and $\mu(\epsilon))[1]$.

\section{LITERATURE REVIEW}

Elasticity imaging methods adopt one concept which is applying either a mechanical excitation or stress to tissues. Stress can be from external or internal excitation source. The internal source may be the physiological motion of the tissue itself or an acoustic radiation force (ARF). The resulting tissue deformation (displacement) is measured in response to that excitation using ultrasound, magnetic resonance or optical methods [1].

Based on the Helmholtz model for shear wave propagation; introduced by equations (3), (4) and (5); the measured tissue deformation can be related to tissue stiffness. When the imaging methods were first proposed, the excitation source was obtained and considered as the physiological pulsation of the tissue itself, and ultrasound was used to monitor the tissue response $[9,10]$. Then, dynamic methods were introduced, where dynamic external vibration is used to create shear waves inside the tissue to be studied (Sonoelasticity) [11] and methods using external static compression for mechanical excitation (strain imaging) [12]. 
The excitation using Acoustic Radiation Force Impulse (ARFI) was introduced in the early $90 \mathrm{~s}$ by Sugimoto et al. [13]. This method has an advantage of coupling the source of excitation to the organ under study directly, rather than being coupled through intervening tissues. Greenleaf et al. and Parker et al. provide efficient reviews of elasticity imaging methods $[14,15]$. Nyborg et al. $[16,17]$ introduced a model of the tissue to be acting as a viscoelastic fluid in response to the ultrasonic wave propagation, and under plane wave assumptions, the ARFI is given by (6).

$$
F=\frac{2 \alpha I}{C}
$$

where $\alpha(\mathrm{dB} / \mathrm{cm} . \mathrm{MHz})$ is the acoustic absorption coefficient of the tissue, $\mathrm{I}$ is the temporal average intensity of the wave and $\mathrm{C}(\mathrm{m} / \mathrm{sec}$.) is the speed of sound in tissue.

Besides, the contribution of scattering is neglected because the majority of attenuation arises from absorption [17]. The relationship between the depth and the frequency should be considered. The attenuation increases by increasing depth for higher frequencies. As a result, there is an optimal frequency for each depth which depends on the attenuation-frequency tradeoff.

The ARFI can be applied for different temporal duration's methods, such as the quasi-statically, transient method, and harmonic method [1, 8]: the Quasi-static method which proposes that; the excitation pulses are applied on the tissue to reach a steady state response, typically longer than one second; the transient method which proposes that the excitation is applied for a very short duration, typically a temporal impulse, faster than the natural frequency of the tissue associated with the dynamic tissue response; and the Harmonic method which proposes the application of the impulse in a harmonic way; a pulsed manner; to achieve a sinusoidal tissue excitation of one or more frequencies.

Applying a specific stress (force) then measuring the corresponding mechanical response is considered as the most common method for elasticity imaging. By having variations in the duration of the applied force and different measuring methods, a variety of imaging methods have been obtained, each having advantages and drawbacks [18]. A brief about the most common methods is given in the following sub-section.

\section{A. Sonoelasticity}

Harmonic shear waves are generated mechanically by using external actuators in contact with the skin. Doppler or any other imaging technique is used for measurement of wave propagation [19].

Krouskop et al. induced the shear wave inside the muscle tissue of thigh using a motorized actuator placed on the medial side of the thigh. The ultrasound transducer was put on the lateral side of the thigh to measure the wave propagation using Doppler methods [19]. On the other hand, Lerner et al. proposed the use of the acoustic horn to generate the waves in the phantom and the use of colored Doppler for measurement [20]. Shear wave velocities distribution is obtained by Sonoelastography for phantoms, human prostates, and skeletal muscles [21].

\section{B. Acoustic Radiation Force Impulse (ARFI) imaging}

Acoustic radiation force impulse (ARFI) is defined as the force resulting from the momentum transfer from the propagating ultrasound wave through the tissue due to absorption and scattering mechanisms [16]. Displacements in tissue can be generated using a focused force impulse, these displacements (deformations) are relaxed and the tissue returns to its original position by the removal of the force [22].

In response to this focused force, the tissue within the region of excitation (ROE) is deformed and shear waves are generated and propagate away from the ROE. After this short duration excitation is achieved, an ordinary imaging procedure is done to image these waves departure along the transverse direction of the ROE. Along a single line, excitation is accomplished and measurement is made, then, another adjacent line is investigated and so on till the image of tissue response is constructed. The tissue response is characterized by a set of parameters; the peak displacement, and the time the tissue takes to reach the peak displacement and the recovery time [23].

This method has been used for many applications. It is used with phantom tissue imaging [24], imaging thermally induced lesions [25], abdominal imaging of lesions [26], human prostate imaging [27] and imaging of cardiovascular vessels [28, 29].

\section{Transient Elastography}

An external source of vibrations is used to generate waves and to provide a single cycle of low frequency; typically 40-50 Hz. Compressional and shear waves are generated together by this sort of excitation. Fortunately, they are separated from each other by the time lag between them, as the compressional wave is much faster than the shear one [30, 31]. Transient excitations avoid biases caused by the sinusoidal excitations of cylindrical source $[30,31]$.

Motion tracking is the most important part of this method, where cross-correlation is used to locate the time shift between two echo signals, and by knowing the speed of sound in tissue we can estimate how much motion has occurred inside the tissue.

Dutt et al. were pioneers to use mechanical actuation and to obtain a measurement of shear waves using this method [32]. Transient elastography can be used to measure stiffness in phantoms, breast, and skeletal muscle [33].

\section{Shear Wave Elasticity Imaging}

The modulated ultrasound beam that generates acoustic radiation force was not used until Nightengale and Trahey have made their experiments on the proposed theory of Sarvazyan et al [34]. The generated force, when applied to the tissue, generates the shear waves that are detected by any other method. It is used to palpate the tissue but from the inside. Thereby, it has replaced the physicians' finger and is used as a virtual finger. Having high localization of induced strain; due to high attenuation after a few micrometers away from the ROE; was the main difference between this method and any other elasticity imaging method. 
The high absorption feature of these newly generated waves was the most important reason for the feasibility of using it. Then, an induced vibration is possible to be located in a very tiny portion of the tissue, namely in the focal zone.

In this technique, the major drawback is the small value of deformation. Hence, very complex signal processing methods are required to accurately estimate the motion [35-37]. This method has been used for investigation of phantoms, liver, prostate, and cardiac tissue [38, 39].

\section{E. Supersonic Shear Imaging}

In the preceding methods mentioned, the imaging procedure was made along a single line and with single focal point probably the focal point. The supersonic shear imaging involves investigation to be done with multiple focal points for the same line. The focal point is changed axially along the vertical beam with a speed much higher than the propagation speed of the shear wave resulted [40]. The multiple shear waves resulting from many focal locations (axially) constructively interfere to construct a conical shear wave [41].

This results in a Mach cone, where the Mach number of excitation can be adjusted to make the shear wave directionally oriented. It can be used for phantoms, liver, skeletal muscle and breast assessments [42].

It has been noticed that in viscous fluids, applying acoustic radiation force generates acoustic streaming or fluid flow, and this fluid flow has a velocity proportional to the fluid viscosity and the boundary condition. It is worthy to mention that phenomenon as it helped greatly in breast cancer detection and differentiation.

Starritt et al. were the first to investigate this phenomenon of generation of acoustic streaming. Nightingale et al. were the first to use it to differentiate between the fluid filled and solid lesions in the breast. This was achieved by interspersing pushing pulses with Doppler pulses to detect the resulting fluid flow using Doppler techniques [34].

\section{Methodology}

In this search, a FEM is constructed and given the mechanical properties of the Agar-gelatine phantom by a Finite Element Modelling software LISA FEA V8.0. For estimating the mechanical properties of this phantom by ultrasound imaging of the shear wave, a point force is applied for about 0.01 seconds in a transient manner. The resulting shear wave is then tracked by B-mode ultrasound imaging at a high frame rate and its speed is estimated to calculate the stiffness of the phantom; which is a mechanical property of the phantom; using Matlab software version R2010a. Tracking the shear wave propagation off-axis and measuring its velocity is a necessary process to have a quantitative map of elasticity for the tissue under consideration.

Tracking process is performed at several stationary nodes inside the model; where every node gives a curve of its displacement versus time profile from which we estimate the speed of the propagated wave. During its propagation; the shear wave; covers a few meters per second, and a specific frame rate (FR) is needed to appropriately catch the propagating peak leading to a good estimation of the wave's speed. Hence, a frame rate of several kilohertz is needed to have a good estimation for the speed since the conventional ultrasound scanners are not efficient due to their low frame rates; typically reaching about 50 to 60 frames per second [43].

The block diagram of the proposed method is shown in Figure 2.

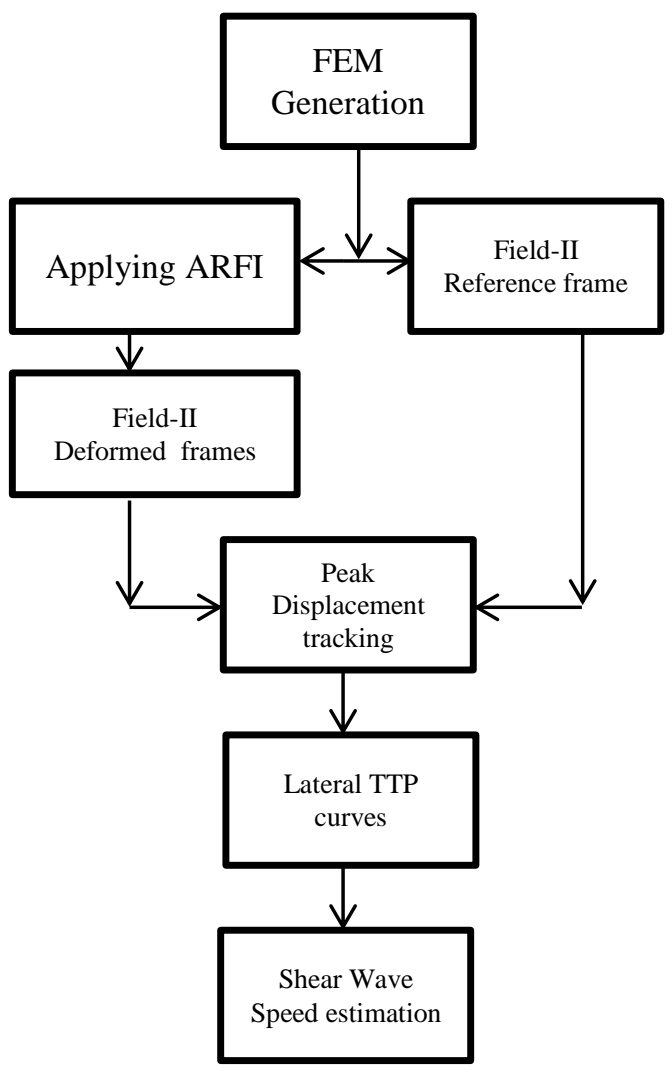

Fig. 2. Block diagram of the methodology

\section{A. FEM Mesh generation}

The experiment is performed using an elastic Agargelatine model. The choice of this material is due to the ability of the gelatine to maintain the stiffness of the phantom, and the ability of the agar to act as scatterers for the ultrasound waves in the phantom. A FEM mesh for this phantom is generated by a Finite Element Modelling (FEM) software LISA FEA V8.0 to simulate its behaviour at applying the acoustic radiation force impulse (ARFI). The FEM is a square shaped plate and unity in dimensions (1m side length), this square shaped plate resembles the plane inside which the ARFI is generated and the shear wave propagation takes place.

Dividing this model to small squares; by Meshing techniques; provides an accurate displacement calculations' leading to a good estimation of the shear wave speed, each sub-square is attached to its neighbourhood by nodes.

It is at these nodes where the wave tracking takes place, this is achieved by tracking the peak displacements using Bmode ultrasound imaging. Differences in times for reaching the peak displacement at two or more successive nodes are calculated to provide the speed estimation. The mesh consists 
of 1105 nodes and 1024 elements, where the opposing face to the transducer is constrained completely and the face where the transducer touches is allowed to move in the perpendicular direction; i.e. the direction of the shear wave and other faces are allowed to move freely in all directions. The distance between any two successive nodes in the lateral direction is 0.015625 meters. The phantom is shown Figure 3.

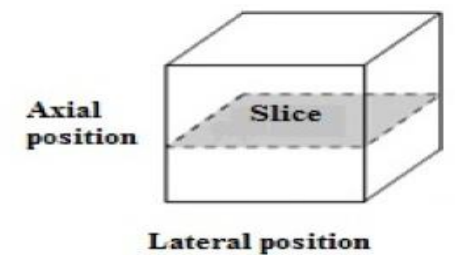

(a)

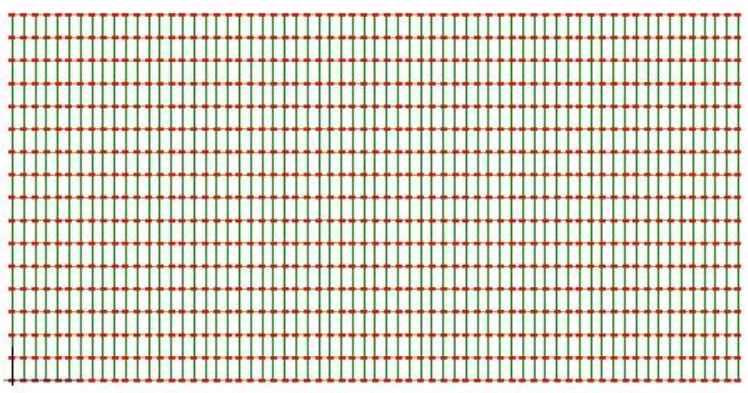

(b)

Fig. 3. Slice of the phantom, and (b) Square shaped $1 \mathrm{~m}$ side length of the Agar-gelatine phantom

Assigning the material mechanical parameters of the Agargelatine phantom for the simulated model to act as a real material, those properties are listed in Table 1.

TABle I. Material Mechanical Parameters for Agar-Gelatine PHANTOM

\begin{tabular}{|l|l|l|}
\hline Material parameter & Value & Unit \\
\hline Young's modulus $\left(E_{o}\right)$ & 107585 & $\mathrm{~Pa}$ \\
\hline Shear modulus $\left(G_{o}\right)$ & 35886 & $\mathrm{~Pa}$ \\
\hline Poisson's ratio $\left(v_{o}\right)$ & 0.499 & --- \\
\hline Density $(\rho)$ & 1060 & $\mathrm{Kg} / \mathrm{m}^{3}$ \\
\hline
\end{tabular}

\section{B. Acoustic Radiation Force Impulse (ARFI) generation}

After generating the mesh nodes, the ARFI is generated and applied on the model. A point source force is generated by LISA FEA V8.0 program and applied at the centre of the mesh model; equally at the focal point of the ultrasound beam in the axial direction; this force is applied for a very short duration, typically 0.02 second and less. The force is applied in a gradually increasing manner, and is removed in a gradually decreasing manner as well; each part of the applying and the removal of the force take typically 0.01 second; as shown clearly in Figure 4.

The time of investigation is 50 milliseconds; the force is induced within 10 milliseconds to reach its peak, and another 10 milliseconds to be fully removed and to reach zero magnitude, summing up for 20 milliseconds of excitation. The rest of the time is for the tracking B-mode imaging procedure.

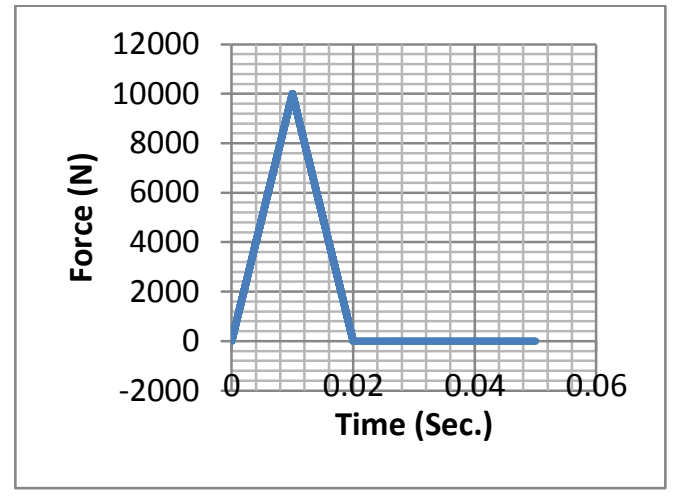

Fig. 4. Force magnitude versus the time of excitation

\section{Acquisition Sequence}

In the beginning, the medium is investigated by using a plane wave. This results in providing a reference frame. Then, the pushing sequence is sent by focusing the ultrasound beam to the area of interest (the focal zone of the beam is the area of interest) for a very short duration, typically about 20 milliseconds.

Just after the generation of the pushing pulse, an ordinary B-mode imaging procedure is carried out to catch the progress of the wave propagation through the model [43]. The B-mode imaging of the shear wave propagation is carried out by a very high frame rate, almost 1000 frames per second and up [43]. For investigating any other region in the model, the last procedure is repeated after modifying the focal zone of the beam.

For visualization of the propagation of the wave, the peak of the wave is plotted versus time profile for the whole model. The speed can be estimated from this visualization. Converting 3D visualizations to $2 \mathrm{D}$ curves of nodes displacements versus time profiles is more efficient for estimating the speed of the shear wave as proposed in [44]. For more feasibility of the calculations, one of the sides of the curve is selected to calculate the shear wave speed from it, by tracking the peak displacement. This is proposed by Ned C. Rouze in [45] and shown in Figure 5.

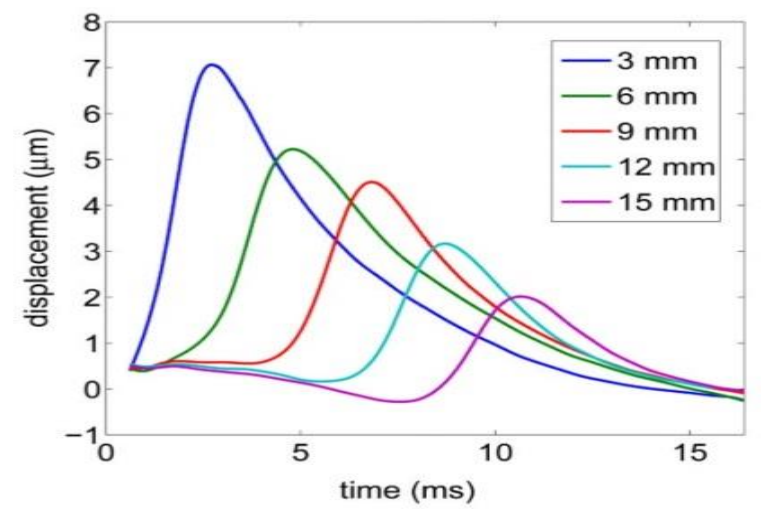

Fig. 5. Single sided curve for estimating the shear wave speed at different lateral positions along the time profile [45] 


\section{RESULTS}

In this section, results for the Agar-gelatine phantom are reported for mechanical properties presented in Table 1. Results are obtained by using both the Finite Element Analysis software LISA in conjunction with Matlab software.

The shear wave speed is estimated using the Time-ToPeak displacement (TTP) method. Time-To-Peak displacement is defined as the time taken by a specific part of tissue to reach its maximum displacement. It is a characteristic for each tissue. Simply, the shear wave speed is estimated as the distance difference between two nodes divided by the time difference at which the TTPs are occurring for these two nodes. Yet, for a good estimation of the shear wave speed, more nodes are involved in the calculation where the average is calculated.

For calculating the TTP, the displacement profile for each node with time must be obtained. In other words, to observe the specific time at which the node reaches its peak displacement. The displacement profile for the central node of excitation is obtained as shown in Figure 6. It is clear from the figure that at $\mathrm{t}=0.014 \mathrm{sec}$. the maximum displacement has taken place. The force is applied at the central node.

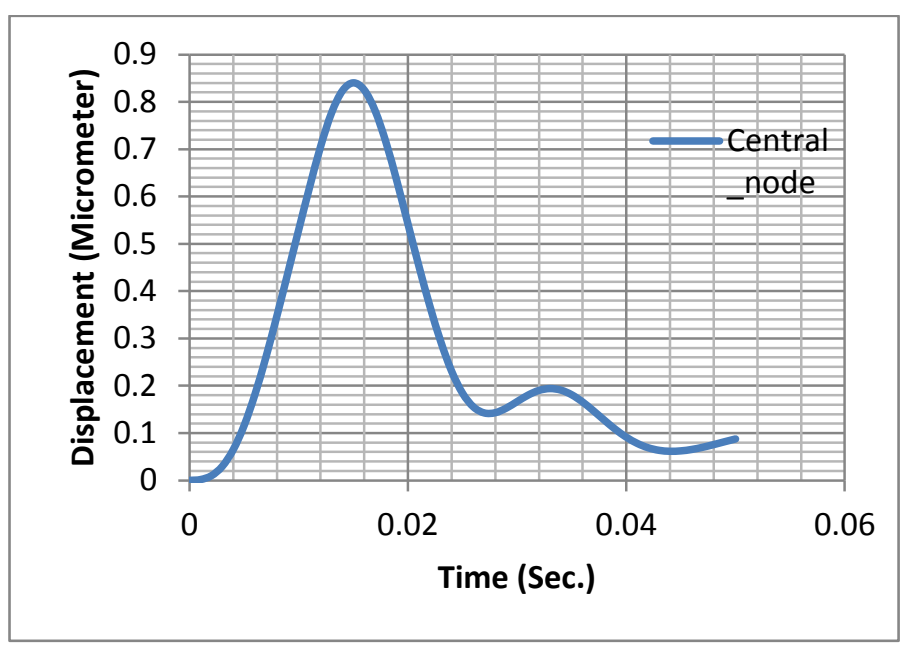

Fig. 6. Displacement magnitude profile

Having displacement profiles for the nodes that are laterally away from the node at which the excitation happens, allows precise estimation of the shear wave speed, by calculating their successive TTPs.

Figure 7 shows clearly the displacements profiles for five successive nodes away from the central node, where the TTPs are calculated from them. Moving outwards from the central node, peaks are observed to be gradually decreasing as the node gets farther.

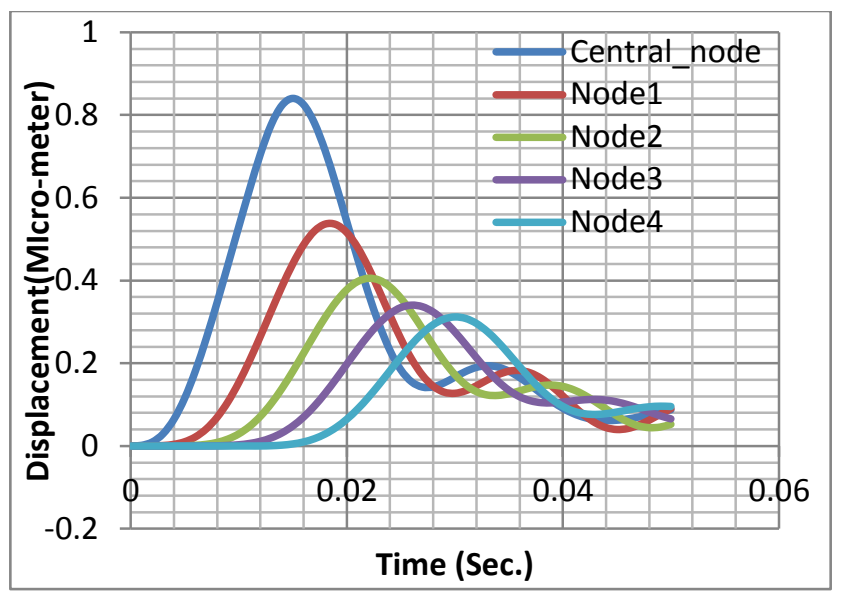

Fig. 7. Displacements profiles for five successive nodes away from the central node

The shear wave speed is calculated as the average value of the quotients, of distance difference between two successive nodes divided by the difference in the TTPs for these same two nodes, for eight nodes, and given by (7) and (8).

$$
\begin{aligned}
& C_{2,1}=\frac{\Delta x}{\Delta t} \\
& C_{\text {avg }}=\frac{\sum_{i=1}^{n}\left(C_{n, n-1}\right)}{n}
\end{aligned}
$$

The following tables (2), (3), and (4) show different obtained values for the shear wave speeds at each successive two nodes. The average speed is found to be $5.2083(\mathrm{~m} / \mathrm{sec})$ at its optimum case of 0.001 seconds as a time difference between each two successive frames.

TABLE I. ShEAR WAVE SPEEDS AT 8 DifFERENT Nodes AT 0.001 SeC DIFFERENCE BETWEEN EACH TWO SUCCESSIVE FRAMES

\begin{tabular}{|l|l|l|l|}
\hline Node no. & $\Delta \mathbf{x}(\mathbf{m})$ & $\Delta \mathbf{t}(\mathbf{s e c})$. & $\mathbf{C}(\mathbf{m} / \mathbf{s e c})$. \\
\hline 0,1 & 0.015625 & 0.003 & 5.2083 \\
\hline 1,2 & 0.015625 & 0.003 & 5.2083 \\
\hline 2,3 & 0.015625 & 0.003 & 5.2083 \\
\hline 3,4 & 0.015625 & 0.003 & 5.2083 \\
\hline 4,5 & 0.015625 & 0.003 & 5.2083 \\
\hline 5,6 & 0.015625 & 0.003 & 5.2083 \\
\hline 6,7 & 0.015625 & 0.003 & 5.2083 \\
\hline
\end{tabular}

TABLE II. SHEAR WAVE SPEEDS AT 8 DifFERENT NODES AT 0.0009 SEC DIFFERENCE BETWEEN EACH TwO SUCCESSIVE FRAMES

\begin{tabular}{|l|l|l|l|}
\hline Node no. & $\Delta \mathbf{x}(\mathbf{m})$ & $\Delta \mathbf{t}(\mathbf{s e c})$. & $\mathbf{C}(\mathbf{m} / \mathbf{s e c})$. \\
\hline 0,1 & 0.015625 & 0.003 & 5.2083 \\
\hline 1,2 & 0.015625 & 0.004 & 3.9063 \\
\hline 2,3 & 0.015625 & 0.004 & 3.9063 \\
\hline 3,4 & 0.015625 & 0.004 & 3.9063 \\
\hline 4,5 & 0.015625 & 0.004 & 3.9063 \\
\hline 5,6 & 0.015625 & 0.003 & 5.2083 \\
\hline 6,7 & 0.015625 & 0.003 & 5.2083 \\
\hline
\end{tabular}


TABLE III. SHEAR WAVE SPEEDS AT 8 DIFFERENT NodES AT 0.0005 SEC DIFFERENCE BETWEEN EACH Two SUCCESSIVE FRAMES

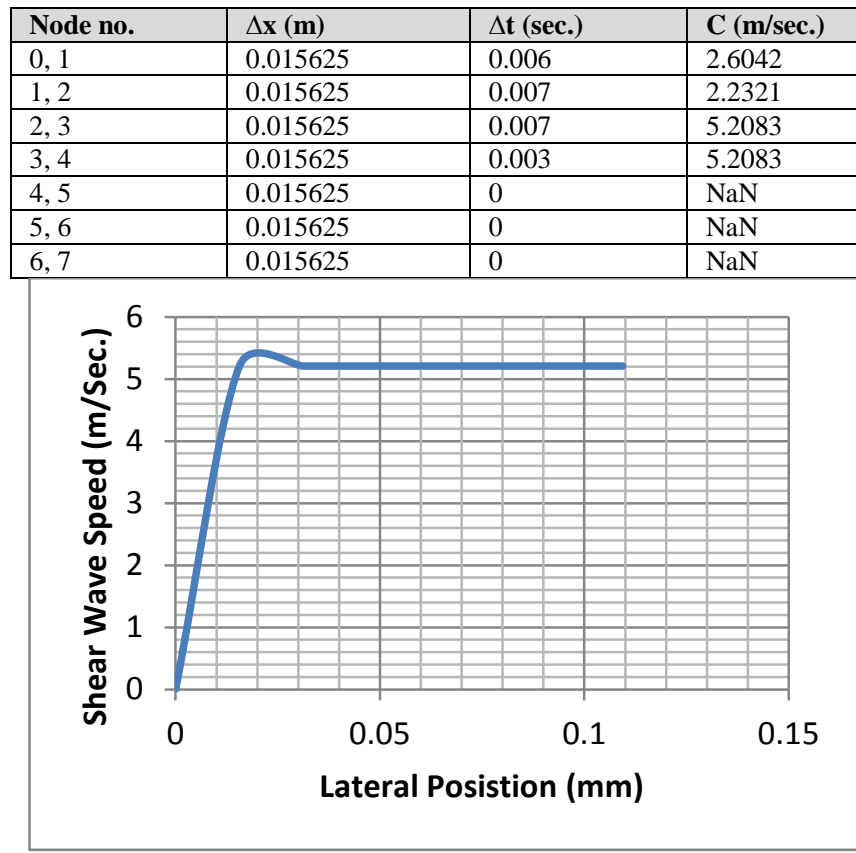

(a)

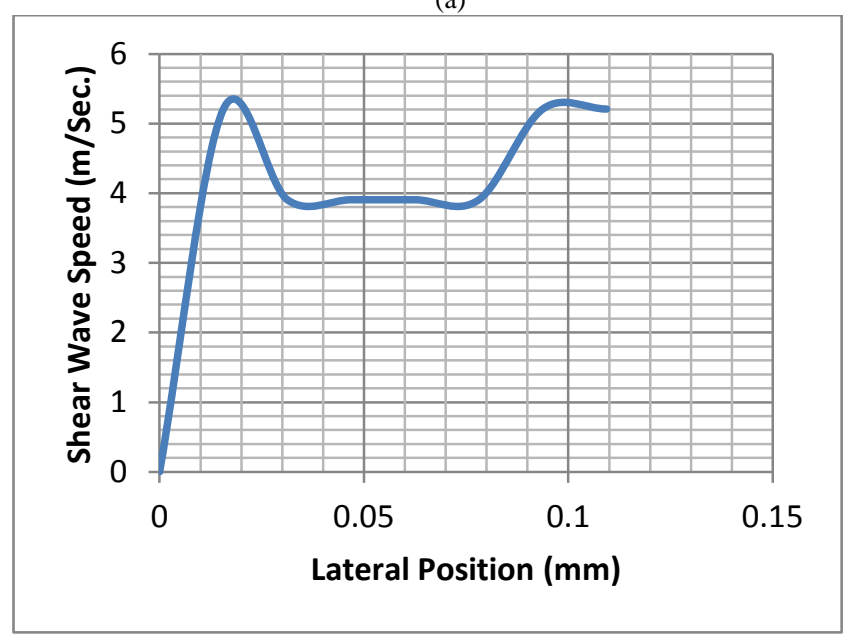

(b)

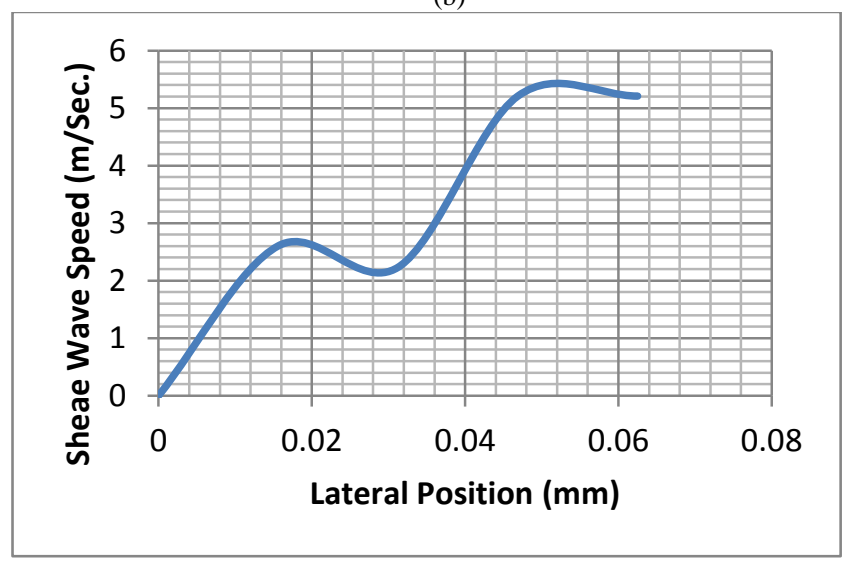

(c)

Fig. 8. Laterally probed velocities at different frame rates: (a) at $1 \mathrm{KHz}$, (b) at $1.1 \mathrm{KHz}$, and (c) at $2 \mathrm{KHz}$

\section{DISCUSSION}

From previous tables, it is clear that estimating the shear wave speed does not give a fixed value for the speed. On other words, there is a tradeoff between the estimated speed and the frame rate used for the estimation process. There is an optimum frame rate that leads to the estimation of the closest speed value, although higher frame rates give closest estimated value.

In our experiments, the optimum frame rate is found to be $1 \mathrm{KHz}$ (each frame of imaging takes about $0.001 \mathrm{sec}$ ). Other frame rates were found to give fluctuating velocities around 5 $\mathrm{m} / \mathrm{seconds}$ as shown in Figure 8. The optimum frame rate in these experiments led to a velocity of $5.2 \mathrm{~m} / \mathrm{sec}$. This is a characteristic for this phantom.

On the first laterally probed velocities curve, it is observed that velocities are fixed and independent on the node lateral position inside the phantom. Other laterally probed velocities curves show the instability of the speed and its dependency on the node lateral position.

The calculated shear wave speed for the phantom under study is about $5.7 \mathrm{~m} / \mathrm{sec}$. The deviation of our results from the calculated is due to the insufficient number of nodes involved in the phantom construction, yet the speed estimation does not give a precise value as the calculated one. Moreover, the distance between the nodes in the $\mathrm{x}$-direction is predicted to have a role in a good estimation of the speed. As the nodes are the stationary stations from which we monitor the propagation of the wave, less distance means better tracking for the peak displacement.

All methods using external actuators and external vibrating sources are introducing a proper deformation value, which is large enough to be picked up and processed. This facilitates the measurement process of tissue nonlinearities. This also has the drawback of complex hardware to achieve such a function. Amongst these methods, Sono elasticity and transient elasticity.

On the other hand, methods which utilize ARFI have the advantage of using the same ultrasound scanner for excitation and imaging, i.e. the same scanner for probing and measurement. The only disadvantage, here, is that the intensity of the excitation pulse must be kept under certain limit due to the mechanical and thermal considerations when dealing with ultrasound waves [46].

These unwanted bio-effects limits the intensity of excitation, and hence the induced deformation to less than 30 $\mu \mathrm{m}$. This also limits the shear wave's estimation beyond 6 or 7 $\mathrm{cm}$, due to high attenuation. Furthermore, as mentioned earlier, the high attenuation is considered an advantage, where it produces highly localized shear waves [46].

As far as we know, there is no formula to predict the relationship between the excitation frequency and the elasticity moduli. If we take into consideration that the tissue particles may be modeled as a vibrating pendulum, there will be some excitation frequency that will have no action on these particles, as their moment of inertia will be very high for that frequency. In other words, shear waves will not be generated 
and hence the tissue will be misestimated to having low modulus of elasticity, but, in reality it is not. This critical frequency or any frequencies approaching it should not be used as they deflect the estimation. This critical excitation frequency will be investigated in our future work.

\section{CONCLUSION}

In this paper, shear wave speed is estimated for the Agargelatine. A phantom with specific mechanical properties from literature is used. A model for this phantom is generated by a finite element modeling software to simulate its behavior. A point source force is applied at the focal point, which is the center of the phantom, to stimulate the shear wave propagation. TTP method is used to estimate the speed. The estimated speed is found to be $5.2 \mathrm{~m} / \mathrm{sec}$; while the calculated one is $5.7 \mathrm{~m} / \mathrm{sec}$. The difference between both of them arises from the shortage of the number of nodes by which the model has been constructed. Further investigation will be carried out to improve the results and to study the effect of the excitation frequency on the estimated speed.

\section{REFERENCES}

[1] M. L. Palmeri, K. R. Nightingale, "Acoustic Radiation Force Based Elasticity Imaging methods", Interfocus, vol. 1, pp. 553-564, 2011.

[2] W. M. Lai, D. Rubin, E.Krempl, "Introduction To Continuum Mechanics”, M.A. Woburn: Butterworth- Heinmann, 1999.

[3] F. Duck, "Physical Properties Of Tissue a Comprehensive Reference Book", New York, NY: Academic Press, 1990.

[4] A. Sarvazyan, A. Skovoroda, S. Emelianov, J. B. Fowlkes, J. G. Pipe, R. S. Adler, R. B. Buxton, L. Carson, "Biophysical Bases Of Elasticity Imaging”, Acoust. Imag., vol.21, 223-240, 1995.

[5] A. Sarvazyan, "Elastic Properties Of Soft Tissue, Handbook Of Elastic Properties Of Solids, Liquids and Gases", eds M. Levy, H. E. Bass and R. R. Stern, London, UK: Academic Press, pp. 107-127, 2001.

[6] C. Kasai, N. Koroku, A. Koyano, R. Omoto, "Real-time Twodimensional Blood Flow Imaging Using an Autocorrelation Technique", IEEE Trans. Ultrason., Ferroelec., Freq. Contr., vol. SU-32, pp. 458463, 1985.

[7] W. Walker, G. Trahey, "A Fundamental Limit On Delay Estimation Using Partially Correlated Speckle Signals", IEEE Trans. Ultrason. Ferroelec. Freq. Contr., vol. 42, pp. 301-308, 1995.

[8] Y. C. Fung, "Biomechanics: Mechanical Properties Of Living Tissues", New York, NY: Springer, 2nd edn. 1993.

[9] R. Dickinson, C. Hill, "Measurement Of Soft Tissue Motion Using Correlation Between A-scans", Ultrasound Med. Biol., vol. 8,pp. 263271, 1982.

[10] L. Wilson, D. Robinson, 'Ultrasonic Measurement Of Small Displacements and Deformations Of Tissue”, Ultrason. Imag., vol. 4, pp. 71-82, 1982.

[11] R. M. Lerner, K. J. Parker, J. Holen, R. Gramiak, R. C. Waag, "Sonoelasticity: Medical Elasticity Images Derived From Ultrasound Signals in Mechanically Vibrated Targets", Acoust. Imag., vol. 16, pp. 317327, 1988.

[12] J. Ophir, I. Cespedes, H. Ponnekanti, X. Li, "Elastography: A Quantitative Method For Imaging The Elasticity Of Biological Tissues", Ultrason. Imag., vol. 13, pp. 111-134, 1991.

[13] T. Sugimoto, S. Ueha, K. Itoh, "Tissue Hardness Measurement Using The Radiation Force Of Focused Ultrasound", IEEEXplore, vol. 3, pp. 1377-1380, 1990

[14] J. F. Greenleaf, M. Fatemi, M. Insana, "Selected Methods For Imaging Elastic Properties Of Biological Tissues”, Ann. Rev. Biomed. Eng., vol. 5, pp. 57-78, 2003.

[15] K. J. Parker, L. S. Taylor, S. M. Gracewski, D. J. Rubens, “A Unified View Of Imaging The Elastic Properties Of Tissue", J. Acoust. Soc. Am., vol. 117, pp. 2705-2712, 2005.
[16] W. L. M. Nyborg, "Acoustic Streaming", In Physical acoustics (ed. W. P. Mason), pp. 265-331, New York, NY: Academic Press Inc, 1965.

[17] G. R. Torr, "The Acoustic Radiation Force", Am. J. Phys., vol. 52, pp. 402-408, 1984.

[18] A. Sarvazyan, T. J. Hall , M. W. Urban, M. Fatemi, S. R. Aglyamov, B. S. Garra, "An Overview Of Elastography, An Emerging Branch Of Medical Imaging", Curr. Med. Imaging Rev., vol. 7, pp. 255-282, 2011.

[19] T. A. Krouskop, D. R. Dougherty, F. S. Vinson, "A Pulsed Doppler Ultrasonic System For Making Noninvasive Measurements Of The Mechanical Properties Of Soft Tissue", J Rehabil Res Dev., vol. 24, pp. $1-8,1987$.

[20] R. M. Lerner, K. J. Parker, J. Holen, R. Gramiak, R. C. Waag, "Sonoelasticity: Medical Elasticity Images Derived From Ultrasound Signals In Mechanically Vibrated Targets"; Acoustical Imaging., vol. 16, pp. 317-327. New York, NY: Plenum Press; 1988.

[21] K. Hoyt, T. Kneezel, B. Castaneda, K. J. Parker, "Quantitative Sonoelastography For The In Vivo Assessment Of Skeletal Muscle Viscoelasticity", Phys Med Biol., vol. 53, pp. 4063-4080, 2008.

[22] K. R. Nightingale, M. L. Palmeri, R. W. Nightingale, G. E. Trahey , "On The Feasibility Of Remote Palpation Using Acoustic Radiation Force", J Acoust Soc Am., vol. 110, pp. 625-34, 2001.

[23] M. L. Palmeri, S. A. McAleavey, K. L. Fong, G. E. Trahey, K. R. Nightingale, "Dynamic Mechanical Response Of Elastic Spherical Inclusions To Impulsive Acoustic Radiation Force Excitation", IEEE Trans Ultrason Ferroelectr Freq Control., vol. 53, pp. 2065-79, 2006.

[24] M. L. Palmeri, S. A. McAleavey, K. L. Fong, G. E. Trahey and K. R. Nightingale, "Dynamic Mechanical Response Of Elastic Spherical Inclusions To Impulsive Acoustic Radiation Force Excitation", IEEE Trans Ultrason Ferroelectr Freq Control., vol. 53, pp. 2065-79, 2006.

[25] B. J. Fahey, K. R. Nightingale, D. L. Stutz, G. E. Trahey, "Acoustic Radiation Force Impulse Imaging Of Thermally- and Chemicallyinduced Lesions In Soft Tissues", preliminary ex vivo results., vol. 30, pp. 321-8, 2004.

[26] B. J. Fahey, K. R. Nightingale, R. C. Nelson, M. L. Palmeri, G. E. Trahey, "Acoustic Radiation Force Impulse Imaging Of The Abdomen: Demonstration Of Feasibility and Utility", Ultrasound Med Biol., vol. 31, pp. 1185-98, 2005.

[27] L. Zhai, J. Madden, W. C. Foo, M. L. Palmeri, V. Mouraviev, T. J. Polascik, K. R. Nightingale, "Acoustic Radiation Force Impulse Imaging Of Human Prostates E-vivo", Ultrasound Med Biol., vol. 36, pp. 576588,2010

[28] D. Dumont, J. Dahl, E. Miller, J. Allen, B. J. Fahey, G. E. Trahey, "Lower-limb Vascular Imaging With Acoustic Radiation Force Elastography: Demonstration Of In-vivo Feasibility", IEEE Trans Ultrason Ferroelectr Freq Control., vol. 56, pp. 931-944, 2009.

[29] J. J. Dahl, D. M. Dumont, J. D. Allen, E. M. Miller, G. E. Trahey, "Acoustic Radiation Force Impulse Imaging For Noninvasive Characterization Of Carotid Artery Atherosclerotic Plaques: A Feasibility Study", Ultrasound Med Biol., vol. 35, pp. 707-716, 2009.

[30] S. Catheline, J. L. Thomas, F. Wu, M. A. Fink, "Diffraction field Of A Low Frequency Vibrator In Soft Tissues Using Transient Elastography", IEEE Trans Ultrason Ferroelectr Freq Control., vol. 46, pp. 1013-1019, 1999.

[31] S. Catheline, F. Wu, M. A. Fink, "A Solution To Diffraction Biases In Sonoelasticity: The Acoustic Impulse Technique", J Acoust Soc Am., vol. 105, pp. 2941-50, 1999.

[32] V. Dutt, R. R. Kinnick, R. Muthupillai, T. E. Oliphant, R. L. Ehman, J. F. Greenleaf, "Acoustic Shear-wave Imaging Using Echo Ultrasound Compared To Magnetic Resonance Elastography", Ultrasound in Med. \& Biol ,vol. 26, pp. 397-403, 2000.

[33] J. L. Gennisson, G. Cloutier, "Sol-gel Transition In Agar-gelatin Mixtures Studied With Transient Elastography", IEEE Trans Ultrason Ferroelectr Freq Control., vol. 53, pp. 716-723, 2006.

[34] K. Nightingale, S. McAleavey, G. E. Trahey, "Shear-wave Generation Using Acoustic Radiation Force: In-vivo and Ex-vivo Results", Ultrasound Med Biol., vol. 29, pp. 1715-23, 2003.

[35] M. A. Lubinski, S. Y. Emelianov, M. O’Donnell, “ Speckle Tracking Methods For Ultrasonic Elasticity Imaging Using Short-time 
Correlation", IEEE Trans Ultrason Ferroelectr Freq Control., vol. 46, pp. 82-96, 1999.

[36] Y. Zheng, S. Chen, W. Tan, R. Kinnick, J. F. Greenleaf, "Detection Of Tissue Harmonic Motion Induced By Ultrasonic Radiation Force Using Pulse-echo Ultrasound and Kalman Filter", IEEE Trans Ultrason Ferroelectr Freq Control., vol. 54, pp. 290-300, 2007.

[37] G. F. Pinton, J. J. Dahl, G. E. Trahey, "Rapid Tracking Of Small Displacements With Ultrasound", IEEE Trans Ultrason Ferroelectr Freq Control., vol. 53, pp. 1103-17, 2006.

[38] M. L. Palmeri, M. H. Wang, J. J. Dahl, K. D. Frinkley, K. R Nightingale, " Quantifying Hepatic Shear Modulus In-vivo Using Acoustic Radiation Force", Ultrasound Med Biol., vol. 34, pp. 546-558, 2008.

[39] R. R. Bouchard, S. J. Hsu, P. D. Wolf, G. E. Trahey, "In-vivo Cardiac, Acoustic-Radiation-Force-Driven, Shear Wave Velocimetry", Ultrason Imaging., vol. 31, pp. 201-213, 2009.

[40] J. Bercoff, M. Tanter, M. Fink, "Sonic Boom In Soft Materials: The Elastic Cerenkov Effect”, Appl Phys Lett., vol. 84, pp. 2202-2204, 2004.

[41] J. Bercoff, M. Tanter, M. Fink, "Supersonic Shear Imaging: A New Technique For Soft Tissue Elasticity Mapping", IEEE Trans Ultrason Ferroelectr Freq Control., vol. 51, pp. 396-409, 2004.
[42] M. Tanter, J. Bercoff, A. Athanasiou, T. Deffieux, J. L. Gennisson, G. Montaldo, M. Muller, A. Tardivon, M. Fink, "Quantitative Assessment Of Breast Lesion Viscoelasticity: Initial Clinical Results Using Supersonic Shear Imaging", Ultrasound Med Biol., vol. 34, pp. 13731386, 2008.

[43] J. Bercoff, M .Tanter, M. Fink, "Supersonic Shear Imaging: A New Technique For Soft Tissue Elasticity Mapping", IEEE Trans Ultrason Ferroelectr Freq Control., vol. 51, pp. 396-409, 2004.

[44] M. L. Palmeri, S. A. McAleavey, K. L. Fong, G. E. Trahey, K. R. Nightingale, "Dynamic Mechanical Response Of Elastic Spherical Inclusions To Impulsive Acoustic Radiation Force Excitation", IEEE Trans Ultrason Ferroelectr Freq Control., vol. 53, pp. 2065-2079, 2006.

[45] N. C. Rouze, M. H. Wang, M. L. Palmeri, K. R. Nightingale, "Robust Estimation Of Time-Of-Flight Shear Wave Speed Using Radon Sum Transform", IEEE Trans Ultrason Ferroelectr Freq Control., vol. 57, pp. 2262-2270, 2010.

[46] A. P. Sarvazyan, O. V. Rudenko, S. D. Swanson, J. B. Fowlkes, S. Y. Emelianov, "Shear Wave Elasticity Imaging: A New Ultrasonic Technology Of Medical Diagnostics", Ultrasound Med Biol., vol. 24, pp. 1419-35, 1998. 